\title{
Modeling for Superheating Phenomenon of Embedded Superfine Metallic Nanoparticles
}

\author{
Hamed Omid ${ }^{1}$, Hamid Reza Madaah Hosseini ${ }^{1,2, *}$ \\ ${ }^{1}$ Department of Materials Science and Engineering, Sharif University of Technology, 14588-9694, Tehran, Iran \\ ${ }^{2}$ Institude for Nanoscience and Nanotechnology, Sharif University of Technology, 14588-9694, Tehran, Iran
}

\begin{abstract}
A theoretical model is proposed to predict the size-dependency of melting point for embedded nanoparticles (NPs) by employing surface and interior average coordination number, cohesive energy and atomic bond strength. The model was applied on the perfect clusters of icosahedral (IC) and body centered tetragonal (BCT) without any vacancies and defects. The predicted results for superfine NPs (lower than $50 \mathrm{~nm}$ ) of $\mathrm{In}, \mathrm{Ag}, \mathrm{Sn}$ and $\mathrm{Pb}$ were consistent with experimental results.
\end{abstract}

Keywords Superheating, Embedded Nanoparticles, Cohesive Energy, Average Coordination Number

\section{Introduction}

Thermodynamic properties which are essential in utilizing nanomaterials in some fields such as microelectronics, nonlinear optics and solar energy, have garnered considerable attentions. Among these properties, melting point is the most practical property in NPs' applications. It is obvious that melting point of NPs is size-dependent, thanks to a lot of works in this field. Melting point decreases with decreasing size for freestanding NPs[1-3]. However, in embedded NPs there are more complexities and the melting point is not only related to NPs' size, structure and shape; but also affected by the embedding matrix. For some matrixes, melting of the embedded NPs occurs in lower temperature than its bulk state, while it is possible for the same NPs to have superheating above the melting point in some other matrices[4-11].

Some experimental results have presented by Sheng et al. $[7,8]$ to reveal increasing or decreasing of melting point for the embedded NPs using differential scanning calorimetry (DSC). The quantity of superheating depends on the coordination of epitaxial interface of the NPs and the embedding matrix. It means if the interface is coherent or semi-coherent, melting point enhances; otherwise, the melting point declines.

Grabaek et al.[4] have acquired some experimental data by plotting melting and solidification thermal hysteresis of small crystalline precipitates of lead in aluminum. According to their report, superheating as well as supercooling is

* Corresponding author:

madaah@sharif.edu (Hamid Reza Madaah Hosseini)

Published online at http://journal.sapub.org/nn

Copyright (C) 2011 Scientific \& Academic Publishing. All Rights Reserved an inherent physical phenomenon.

Jiang et al.[5,6] have developed a model for the superheating behavior of NPs embedded in a matrix, based on the size-dependency of the atomic vibrations amplitudes and the Lindemann criterion. According to their model, superheating can happen if the atomic diameter of the matrix component is smaller than the atomic diameter of the NPs.

There are a few modeling methods or experimental results on the superheating phenomenon[12-14] rather than depression of melting point. Generally, interfaces energy, surface energy and atomic vibrations amplitudes have been employed to model the melting point of embedded NPs. In the present work, modeling is performed by surface $\left(C_{\sigma}\right)$ and interior $\left(C_{i}\right)$ average coordination number of metallic clusters, cohesive energy $\left(E_{C}\right)$, and the ratio of atomic bond strength $(\varphi)$. It is shown that the proposed model would be able to predict the melting point of embedded NPs $\left(T_{m}^{P}\right)$ as a function of their sizes. The results are compared with experimental data.

\section{Theoretical Model}

The cohesive energy of bulk $\left(E_{C}^{b}\right)$ could be obtained as:

$$
E_{C}^{b}=\frac{\varepsilon}{2} C_{i} N
$$

Where $\varepsilon$ is the bond strength and $N$ is total number of cluster atoms which consists of the interior $\left(N_{i}\right)$ and the surface $\left(N_{\sigma}\right)$ atoms.

The cohesive energy for a freestanding NP at previous works was obtained as follows[15,16]:

$$
E_{C}^{P}=\frac{1}{2} \varepsilon\left(C_{i} N_{i}+C_{\sigma} N_{\sigma}\right)
$$


Since embedded NPs have not dangling bond, the rest of surface coordination number $\left(C_{i}-C_{\sigma}\right)$ make new bonds with the embedding matrix. The energy of this new bond is $\varepsilon^{\prime}$. Therefore cohesive energy of embedded NPs can be expressed by:

$$
E_{C}^{P}=\frac{1}{2} \varepsilon\left(C_{i} N_{i}+C_{\sigma} N_{\sigma}\right)+\alpha \varepsilon^{\prime}\left(C_{i}-C_{\sigma}\right) N_{\sigma}
$$

Referring to Eq. (1) and Eq. (3) the following equation can be derived:

$$
E_{C}^{P}=E_{C}^{b}\left(1-\mu \cdot \frac{C_{i}-C_{\sigma}}{C_{i}} \cdot \frac{N_{\sigma}}{N}\right)+2 E_{C}^{b} \alpha \varphi \mu\left(\frac{C_{i}-C_{\sigma}}{C_{i}}\right) \cdot \frac{N_{\sigma}}{N}
$$

Where $\varphi$ denotes the ratio of $\varepsilon^{\prime} / \varepsilon, \mu$ is shape factor[17] and $\alpha[18]$ is the correlation number between atoms of the NPs and those of the surrounding matrix. When $\alpha=0$ the Eq. (4) could be used for freestanding NPs and $\alpha=1$ is for coherent interface between embedded NPs and host matrix.

After simplifying of Eq. (4), the proportion of the NPs' cohesive energy to the bulk state can be obtained as:

$$
\frac{E_{C}^{P}}{E_{C}^{b}}=1-(1-2 \alpha \varphi) \mu \cdot \frac{C_{i}-C_{\sigma}}{C_{i}} \cdot \frac{N_{\sigma}}{N}
$$

Additionally, the relation between cohesive energy and melting point of NPs can be written as[2,19]:

$$
\frac{E_{C}^{P}}{E_{C}^{b}}=\frac{T_{m}^{P}}{T_{m}^{b}}
$$

Eventually, the melting point of NPs can be calculated by combination of Eq. (5) and Eq. (6):

$$
\frac{T_{m}^{P}}{T_{m}^{b}}=1-(1-2 \alpha \varphi) \mu \cdot \frac{C_{i}-C_{\sigma}}{C_{i}} \cdot \frac{N_{\sigma}}{N}
$$

If $2 \alpha \varphi$ is more than one, NPs exhibit superheating above the melting point of corresponding bulk crystal. But in the inert substrates, the tendency of making bonds between the NPs and the substrate $(\alpha)$ decreases. Hence the superheating declines.

Surface $\left(C_{\sigma}\right)$ and interior $\left(C_{i}\right)$ average coordination number, Surface $\left(N_{\sigma}\right)$ and total $(N)$ atoms of a cluster can be calculated by a manner presented in the authors' previous works $[15,16]$. Shape factor for body centered cubic (BCC) and FCC structures have been mentioned in Table 1.

Table 1. Shape Factor for Different Shapes and Lattice Structures

\begin{tabular}{|ccc|}
\hline Structure & Shape & Shape factor $(\mu)$ \\
\hline \multirow{3}{*}{ FCC } & Cubic & 1.654 \\
& Icosahedral & 1 \\
& Sphere & 0.9393 \\
\hline \multirow{2}{*}{ BCC } & Cubic & 1 \\
& Sphere & 0.8060 \\
\hline
\end{tabular}

\section{Results and Discussion}

To compute melting point of NPs based on Eq. (7), the melting point of bulk state and $\varphi$ constant have been summarized in Table 2.

Figure 1 presents a comparison between the Eq. (7) for $\alpha=1$, the models of Nanda et al.[18] and Jiang et al.[5,6], and the experimental results[10] of the superheating behavior of In NPs embedded in $\mathrm{Al}$ matrix. In with $\mathrm{BCT}$ structure was supposed to be BCC for a simplifying purpose. The liquid-drop model of Nanda et al.[18] overestimates the melting point of embedded In NPs in Al matrix. For NPs having diameter higher than $30 \mathrm{~nm}$ the model of Jiang et al.[5,6] has a credible prediction, but for lower than $30 \mathrm{~nm}$ our modeling is more eligible. It can be observed that the whole of models predict increasing melting point with decreasing particle size. This fact could be explained by the ratio of surface to interior atoms which increases with decreasing NP size. The bond energy between NP and matrix atoms $\left(\varepsilon^{\prime}\right)$ is more than the bond energy of NP atoms $(\varepsilon)$ in this case, hence the superheating behavior occurs. In other words, suppression of thermal vibration of atoms on the NPs' interface causes superheating due to coherent interfaces with strong atomic bonds.

The Eq. (7) for $\alpha=0$ has been compared with experimental results of freestanding In NPs[20], and they show good agreement.

Table 2. The Structure, the melting point of bulk state, and $\varphi$ constant

\begin{tabular}{|cccc|}
\hline Element & Structure & $T_{m}^{b}[\mathrm{~K}]$ & $\varphi$ \\
\hline $\mathrm{In}$ & $\mathrm{BCT}$ & 429.75 & 1.17 \\
$\mathrm{Ag}$ & $\mathrm{FCC}$ & 1234 & 1.13 \\
$\mathrm{Sn}$ & $\mathrm{BCT}$ & 505.08 & 0.86 \\
$\mathrm{~Pb}$ & $\mathrm{FCC}$ & 600.61 & 1.27 \\
\hline
\end{tabular}

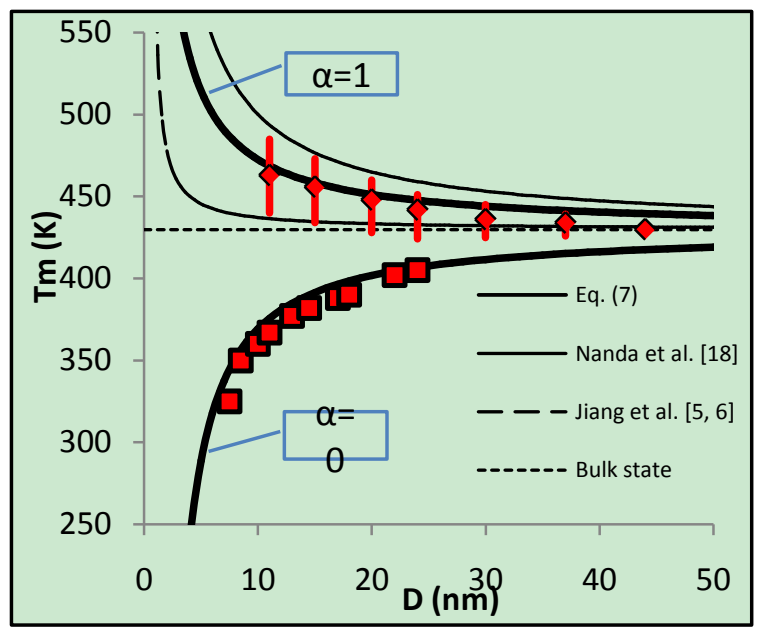

Figure 1. The relation of embedded ( $\alpha=1$ ) and freestanding ( $\alpha=0$ ) In NPs melting point with the size

Figure 2 shows comparison of the three models with experimental results[21] of superheating behavior of Ag NPs embedded in Ni matrix. Ag with FCC structure has been considered as IC clusters. The present model has a good correspondence with the experimental data. Furthermore, Eq. (7) for freestanding NPs has a reliable prediction. The experimental data of Ag freestanding NPs[3] was obtained indirectly by the equation $T_{m}^{P}={ }_{m}^{H} / S_{m}^{P}$, where $H_{m}^{P}$ and $S_{m}^{P}$ are melting enthalpy and entropy of NP, respectively. 


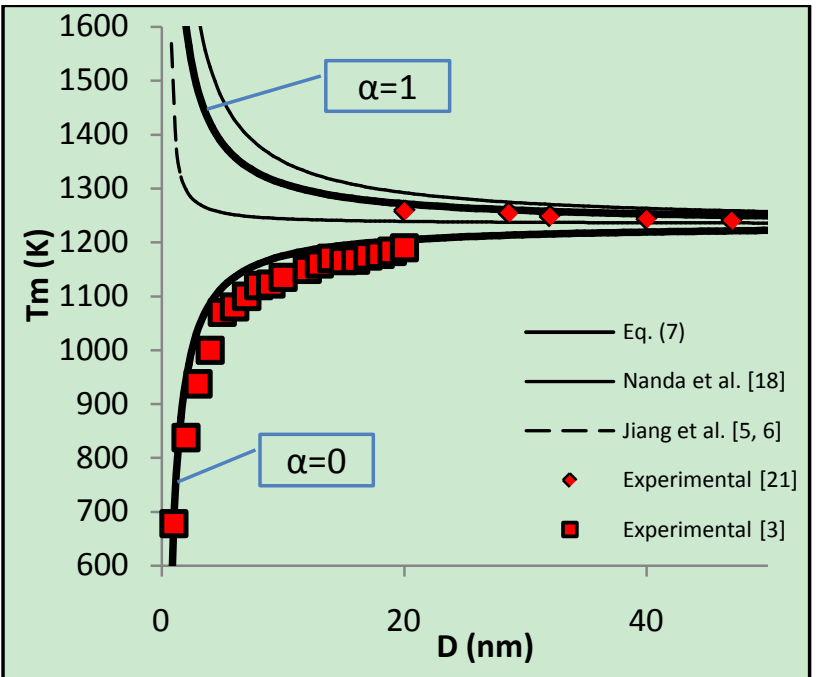

Figure 2. The relation of embedded ( $\alpha=1)$ and freestanding $(\alpha=0)$ Ag NPs melting point with the size

Figure 3 shows that our model predicts the melting point quantity between the model of Nanda et al.[18] and Jiang et al. $[5,6]$ in whole range of NP size. To examine melting point of Sn with BCT structure, it was considered as BCC spherical nanoclusters. Thanks to consider cohesive energy and surface coordination number as size-dependent parameters, the present model exhibits good precision with experimental data[22].

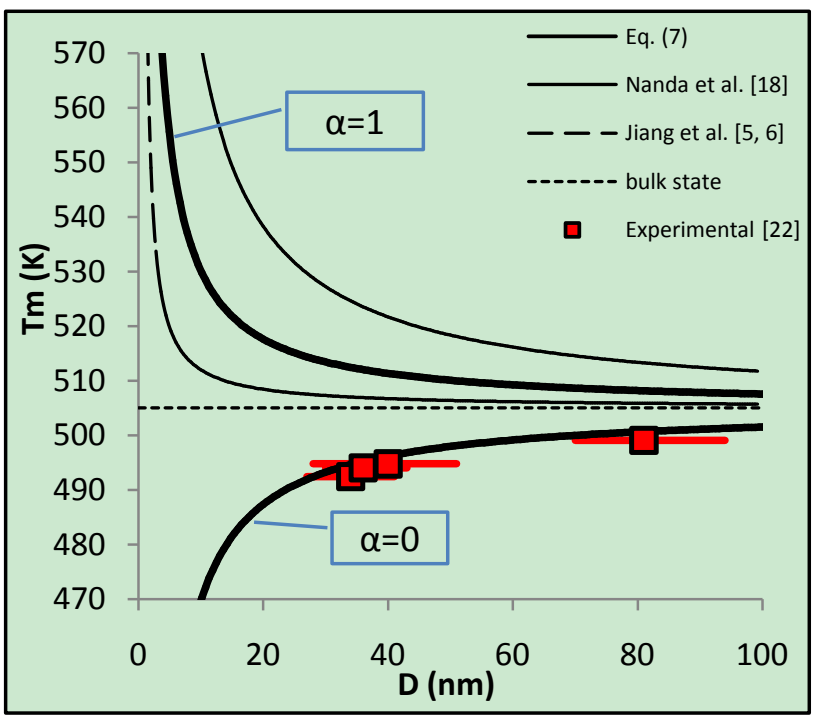

Figure 3. The relation of embedded ( $\alpha=1)$ and freestanding $(\alpha=0)$ Sn NPs melting point with the size

In figure 4 , three models have been compared with three different experimental results[4,8,11]. It could be seen that the different experimental data have not harmony with each other. In superfine particles (lower than $50 \mathrm{~nm}$ ) there is lack of the reliable data for each element, and different methods of experiment reveal different results. Accordingly, experimental results of Graback and Bohr[4] is consistent with the model of Nanda et al.[18], however, work of Sheng[8] is in good accordance with our model. None of these three models follow the experimental results of Chattopadhyay and Goswami[11] precisely.

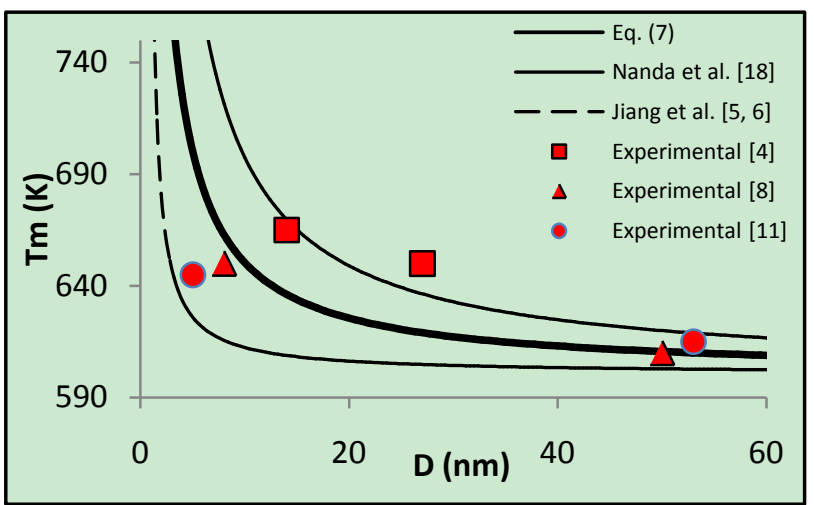

Figure 4. The relation of embedded ( $\alpha=1$ ) $\mathrm{Pb}$ NPs melting point with the size

\section{Conclusions}

It is shown that the simple model based on variable coordination number and cohesive energy can explain the size-dependent melting point of embedded and freestanding NPs as a function of their size, structure and geometry. Moreover, the superheating behavior of embedded NPs in a matrix can be explained by this model. According to the presented model, increasing superheating is due to the bond energy between the cluster's surface atoms and the matrix component atoms $\left(\varepsilon^{\prime}\right)$, which is more than the bond energy between the interior atoms of cluster $(\varepsilon)$. For embedded NPs with coherent interface $(\alpha=1)$ and $\varphi>0.5$, superheating can be observed. The results show this model is in good agreement with experimental data for superheating of embedded NPs in a matrix.

\section{REFERENCES}

[1] M. Takagi, "Electron-diffraction study of liquid-solid transition of thin metal films," Journal of the Physical Society of Japan, vol. 9, pp. 359-363, 1954.

[2] R. Shidpour, H. Delavari H., M. Vossoughi, "Analytical model based on cohesive energy to indicate the edge and corner effects on melting temperature of metallic nanoparticles," Chemical Physics, vol. 378, pp. 14-18, 2010.

[3] W. Luo, W. Hu, S. Xiao, "Size effect on the thermodynamic properties of silver nanoparticles," Journal of Physical Chemistry C, vol. 112, pp. 2359-2369, 2008.

[4] L. Grabaek, J. Bohr, E. Johnson, A. Johansen, L. Sarholt-Kristensen, H.H. Andersen, "Superheating and supercooling of lead precipitates in aluminum," Physical Review Letters, vol. 64, 934-937, 1990.

[5] Q. Jiang, Z. Zhang, J.C. Li, "Superheating of nanocrystals embedded in matrix," Chemical Physics Letters, vol. 322, pp. 549-552, 2000 
[6] Z. Zhang, J.C. Li, Q. Jiang, "Modelling for size-dependent and dimension-dependent melting of nanocrystals," Journal of Physics D: Applied Physics, vol. 33, pp. 2653-2656, 2000.

[7] H.W. Sheng, G. Ren, L.M. Peng, Z.Q. Hu, K. Lu, "Epitaxial dependence of the melting behavior of in nanoparticles embedded in Al matrices," Journal of Materials Research, vol. 12, pp. 119-123, 1997.

[8] H.W. Sheng, G. Ren, L.M. Peng, Z.Q. Hu, K. Lu, "Superheating and melting-point depression of $\mathrm{Pb}$ nanoparticles embedded in Al matrices," Philosophical Magazine Letters, vol. 73, pp. 179-186, 1996.

[9] F.G. Shi, "Size dependent thermal vibrations and melting in nanocrystals," Journal of Materials Research, vol. 9, pp. 1307-1313, 1994.

[10] H. Saka, Y. Nishikawa, T. Imura, "Melting temperature of In particles in an Al matrix," Philosophical Magazine A: Physics of Condensed Matter, Structure, Defects and Mechanical Properties, vol. 57, pp. 895-906, 1988.

[11] K. Chattopadhyay, R. Goswami, "Melting and superheating of metals and alloys," Progress in Materials Science, vol. 42, pp. 287-300, 1997.

[12] H.W. Sheng, K. Lu, E. Ma, "Melting and freezing behavior of embedded nanoparticles in ball-milled Al-10 WT\% M (M = In, $\mathrm{Sn}, \mathrm{Bi}, \mathrm{Cd}, \mathrm{Pb}$ ) mixtures," Acta Materialia, vol. 46, pp. 5195-5205, 1998.

[13] J. Daeges, H. Gleiter, J.H. Perepezko, "Superheating of metal crystals,” Physics Letters A, vol. 119, pp. 79-82, 1986.

[14] C.J. Rossouw, S.E. Donnelly, "Superheating of small solid-argon bubbles in aluminum," Physical Review Letters, vol. 55, pp. 2960, 1985.
[15] H. Delavari H, H. Madaah Hosseini, A. Simchi, "A simple model for the size and shape dependent Curie temperature of freestanding $\mathrm{Ni}$ and $\mathrm{Fe}$ nanoparticles based on the average coordination number and atomic cohesive energy," Chemical Physics In Press, Corrected Proof, 2011.

[16] H. Omid, H. Delavari H, H.R. Madaah Hosseini, "Melting enthalpy and entropy of freestanding metallic nanoparticles based on cohesive energy and average coordination number," Journal of Physical Chemistry C, vol. 115, pp. 17310-17313, 2011.

[17] L.-f. Cao, D. Xie, M.-x. Guo, H.S. Park, T. Fujita, "Size and shape effects on Curie temperature of ferromagnetic nanoparticles," Transactions of Nonferrous Metals Society of China, vol. 17, pp. 1451-1455, 2007.

[18] K.K. Nanda, S.N. Sahu, S.N. Behera, "Liquid-drop model for the size-dependent melting of low-dimensional systems," Physical Review A - Atomic, Molecular, and Optical Physics, vol. 66, pp. 132081-132088, 2002.

[19] G. Guisbiers, "Size-dependent materials properties towards a universal equation," Nanoscale Research Letters, vol. 5, pp. 1132, 2010.

[20] G.L. Allen, R.A. Bayles, W.W. Gile, W.A. Jesser, "Small particle melting of pure metals," Thin Solid Films, vol. 144, pp. 297-308, 1986.

[21] J. Zhong, L.H. Zhang, Z.H. Jin, M.L. Sui, K. Lu, "Superheating of Ag nanoparticles embedded in Ni matrix," Acta Materialia, vol. 49, pp. 2897-2904, 2001.

[22] C.d. Zou, Y.1. Gao, B. Yang, Q.j. Zhai, "Size-dependent melting properties of Sn nanoparticles by chemical reduction synthesis," Transactions of Nonferrous Metals Society of China (English Edition), vol. 20, pp. 248-253, 2010. 